\title{
Evaluation of the optimal standardized ileal digestible threonine:lysine ratio in lactating sow diets
}

\author{
Laura Greiner, ${ }^{\dagger, \hbar, 1}$ Amanda Graham, ${ }^{\dagger, \| l}$ Marcio Goncalves, ${ }^{\$, \uparrow}$ Uiseli Orlando, ${ }^{\$}$ and Kevin J. Touchette**
}

†Carthage Innovative Swine Solutions, LLC, Carthage, IL 62321; Iowa State University, 109 Kildee Hall, Ames,

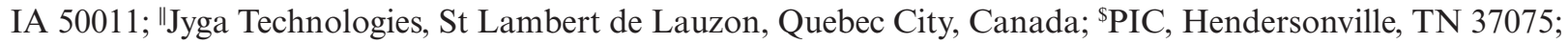

ๆ Jefo, Saint Hyacinthe, Quebec, Canada; and **Ajinomoto Heartland LLC, Chicago, IL 60631

\begin{abstract}
Camborough PIC sows $(n=291)$ were fed 1 of 5 dietary treatments to evaluate the standardized ileal digestible (SID) Thr:Lys requirement during lactation. Sows were blocked by parity $(1,2$, and $3+)$ and randomly allotted to 1 of 5 SID Thr:Lys ratios $(52,60,68,76$, and 84). SID Lys was formulated to $1.03 \%$ and SID methionine and cystine $(\mathrm{M}+\mathrm{C})$ :Lys was $55 \%$. Sows were allowed $5.45 \mathrm{~kg}$ feed/day starting on the day of farrowing. All other nutrients met or exceeded the NRC (2012) requirements. Sows were allowed ad libitum access to water, and piglets were cross-fostered within treatment within $24 \mathrm{~h}$ of birth. Data collected during the study included sow prefarrow BW, sow weaning weight, starting litter weight, weaning litter weight, piglet mortality, weaning-to-estrus interval, and subsequent total born piglets. Data were analyzed as a randomized complete block design using PROC MIXED with sow as the experimental unit and treatment and parity as fixed effects. Requirement
\end{abstract}

estimation models evaluated were quadratic polynomial, broken-line linear, and broken-line quadratic (BLQ) using PROC NLMIXED. Models that differed in their Bayesian information criterion (BIC) values by at least 2 points were considered to have meaningful differences in their data fit. The best-fit model was based on the best BIC value. As the SID Thr:Lys ratio increased, there was a quadratic response on ADFI (4.73, $4.57,4.67,4.55$, and $4.60 \mathrm{~kg} / \mathrm{d} ; P=0.001)$ and percent of sow BW loss $(-6.4,-7.89,-7.35,-6.19$, and $-5.81 ; P=0.004)$. In addition, there was a trend for weaning-to-estrus interval to be affected by the ratio $(5.4,4.4,4.6,4.5$, and $4.8 ; P=0.07)$. Daily litter gain was also quadratically improved as the SID Thr:Lys ratio increased $(2.67,2.67$, $2.78,2.77$, and $2.68 \mathrm{~kg} / \mathrm{d} ; P$-value $=0.001)$. For daily litter gain, the BLQ model was determined to be the best fit with an estimated SID Thr:Lys requirement of $65 \%$. In conclusion, the optimal SID Thr:Lys ratio for litter growth was 65 .

Key words: lactation, litter growth, sow, threonine ratio

(C) The Author(s) 2019. Published by Oxford University Press on behalf of the American Society of Animal Science. All rights reserved. For permissions, please e-mail: journals.permissions@oup.com.

J. Anim. Sci. 2019.97:2972-2978 doi: 10.1093/jas/skz181

\section{INTRODUCTION}

Threonine is an essential AA that is involved in protein deposition, immune function, and gut development. Over the last $12 \mathrm{yr}$, the Danish sow has increased litter size by 3.4 piglets (Nielsen et al., 2018). In the United States, the number of

${ }^{1}$ Corresponding author: greinerl@iastate.edu

Received December 26, 2018.

Accepted May 23, 2019. pigs weaned has increased by 0.5 pigs/sow, and lactation length has increased by $2 \mathrm{~d}$ in the last $7 \mathrm{yr}$ (Stalder, 2018). With the increasing number of pigs nursed and the duration of lactation increasing, the lactating sow has a high protein and energy demand for milk production with $95 \%$ of the indispensable AA requirements to be derived for milk synthesis (NRC, 1998). In addition, the time around farrowing is associated with the highest rate of sow mortality (Deen and Xue, 1999), which indicates that optimal immune function is needed. 
During lactation, when AA quantities are not supported through the feed, the sow must mobilize tissue to support the demands of lactation. The mobilization of tissue (protein mass and backfat) has been estimated from multiple studies into equations presented in the NRC (2012). These calculations are based on predicting the lysine requirement with the ratios of the remaining AAs relative to lysine. While it is assumed that as milk output increases (e.g., increased litter size), that the requirements of AAs relative to lysine remain unchanged, there is limited data available to determine the optimal concentration of Thr in the diet for high producing lactating sows. Furthermore, with the reduction of soybean meal and the use of feed grade lysine in the diet, threonine concentrations are reduced without the use of feed grade AAs. It is hypothesized that the highly prolific sow requires a higher Thr:Lys requirement due to the increased milk production required to support the longer lactation period for piglet protein deposition and mammary/maintenance needs for protein.

Research conducted by Cooper et al. (2001) demonstrated that total threonine requirement of 37 to $40 \mathrm{~g} / \mathrm{d}$ for sow growth and 36 to $39 \mathrm{~g} / \mathrm{d}$ for litter growth. Furthermore, Schneider et al. (2005) estimated a $0.50 \%$ SID threonine requirement (28 g/d) or 57\% Thr:Lys ratio. With little current research to support current requirements in sows with large number of pigs weaned and faster litter growth rate, there is a need for additional evaluation of the Thr:Lys requirement. Therefore, the objective of the study was to determine the Thr:Lys ratio for lactating sow populations that were either gaining or losing weight during lactation.

\section{MATERIALS AND METHODS}

The study was conducted in a commercial 6,000 sow farm located in Western Illinois of the United States. All animal care practices were conducted by following the routine farm management procedures and Pork Quality Assurance guidelines (National Pork Board, 2012). In addition, an internal Carthage Innovative Swine Solutions Animal Care Committee had approved dietary lactation trial protocols with standard measurements and criteria in 2015. The trial protocol complied with the approved standard protocol and was documented into the approved study list for the animal care committee.

\section{Animals}

The study was conducted starting in January through March 2015. Three hundred and thirtythree multiparous and primiparous sows (PIC
Camborough, PIC USA, Hendersonville, TN) were evaluated. Sows were blocked as parity 1 (69), parity 2 (47), or parity $3+(217)$. The sows were porcine reproductive and respiratory syndrome virus (PRRS) negative and Mycoplasma hyopneumonaie stable. The piglets were PRRS and porcine epidemic diarrhea virus negative. Only PRRS antibodies were present from the infection 6 mo prior to the start of the study. In addition, no clinical signs of Mycoplasma hyopneumonaie were present.

\section{Diets}

Diets consisted of corn and soybean meal (Table 1). Diets were held to a constant SID Lys level (1.03\%), and all ratios except for SID Thr:Lys were held constant. Five treatments to be fed to sows were designed with increasing SID Thr:Lys ratios of 46, 52, 58, 64, and 70, respectively. Diets were formulated to be isocaloric (3.2 Mcal ME/kg) and contained vitamins and minerals that exceeded recommendations (NRC, 2012). Energy values for individual ingredients were calculated using the ME values from the NRC (2012).

\section{Lactation Feeding}

Upon entering the farrowing unit at $112 \pm 2 \mathrm{~d}$ of gestation until the time of farrowing, sows were fed $1.8 \mathrm{~kg} / \mathrm{d}$ of the respective lactation diet. After farrowing, sows were fed $1.8 \mathrm{~kg}$ on day $1,2.7 \mathrm{~kg}$ on day $2,3.6 \mathrm{~kg}$ on day 3 of lactation, and then allowed a maximum of $5.45 \mathrm{~kg} / \mathrm{d}$ to provide sows $56 \mathrm{~g}$ of SID Lys intake per day.

Feed was delivered to each sow through the automated Howema Feed System (Big Dutchman, Vechta, Germany). The low (46\%) SID Thr:Lys ratio diet and the high ( $70 \%)$ SID Thr:Lys ratio diet were manufactured at a local feed mill and delivered to the facility. The feed system blended the intermediate diets by delivering a set percentage of each diet into a mixing hopper. The feed system then recorded the actual weight of each diet as it was mixed and then delivered the individual batch of feed to the corresponding sow. At the time of delivery, the system recorded the amount of feed delivered and tracked total lactation consumption per sow. Feed was delivered to each sow via a cable system and was held in a $6.8 \mathrm{~kg}$ plastic hopper (Automated Production Systems, Assumption, IL) attached to an InTak feeder (Automated Production Systems, Assumption, IL). The feed remained in the hopper above the feed pan until the sow moved the InTak feeder actuator causing the feed to fall into the feed 
Table 1. Diet composition of lactation diets for the evaluation of the standardized ileal digestible (SID) Thr:Lys ratio for lactating primiparous and multiparous sows

\begin{tabular}{|c|c|c|}
\hline \multirow[b]{2}{*}{ Ingredient, $\% 0^{1}$} & \multicolumn{2}{|c|}{ SID Thr:Lys ratio } \\
\hline & 52.0 & 84.0 \\
\hline Corn & 72.09 & 71.85 \\
\hline Soybean meal, $48 \%$ & 21.50 & 21.50 \\
\hline Limestone & 1.13 & 1.13 \\
\hline Monocalcium phosphate, $21 \%$ & 1.28 & 1.28 \\
\hline Corn oil & 2.00 & 2.00 \\
\hline Salt & 0.45 & 0.45 \\
\hline L-Lys $\mathrm{HCl}$ & 0.45 & 0.45 \\
\hline L-Thr & - & 0.25 \\
\hline L-Trp & 0.03 & 0.03 \\
\hline $\mathrm{HMTBa}^{2}$ & 0.11 & 0.11 \\
\hline L-Val & 0.32 & 0.32 \\
\hline Feed disinfectant & 0.33 & 0.33 \\
\hline VTM with phytase ${ }^{3}$ & 0.23 & 0.23 \\
\hline Choline chloride & 0.10 & 0.10 \\
\hline \multicolumn{3}{|l|}{ Planned composition } \\
\hline $\mathrm{ME}, \mathrm{Mcal} / \mathrm{kg}^{4}$ & 3.28 & 3.28 \\
\hline $\mathrm{CP}, \%$ & 16.25 & 16.41 \\
\hline $\mathrm{Ca}, \%$ & 0.81 & 0.80 \\
\hline $\mathrm{P}, \%$ & 0.59 & 0.59 \\
\hline Available P, \% & 0.43 & 0.43 \\
\hline Sodium, $\%$ & 0.20 & 0.20 \\
\hline Total Thr & 0.56 & 0.80 \\
\hline SID Lys, $\%$ & 1.03 & 1.03 \\
\hline SID Thr, $\%$ & 0.46 & 0.71 \\
\hline SID M+C:Lys ${ }^{5}$ & 0.55 & 0.55 \\
\hline SID Thr:Lys & 0.46 & 0.70 \\
\hline SID Trp:Lys & 0.18 & 0.18 \\
\hline SID Val:Lys & 0.85 & 0.85 \\
\hline
\end{tabular}

${ }^{1}$ Ingredients are presented as percent inclusion in the diet and are reported on an "as-fed" basis.

${ }^{2} \mathrm{HMTBa}$ - ALIMET is a trademark of Novus International, Inc. and is registered in the United States and other countries. Methionine source. 88\% 2-hydroxy,4-methylthio butanoic acid HMTBa.

${ }^{3}$ Vitamin trace mineral (VTM) premix supplied per kilogram of diet: vitamin A, 13,201 IU; vitamin $\mathrm{D}_{3}, 2,596 \mathrm{IU}$; vitamin $\mathrm{E}, 123.2$ IU; vitamin K (menadione activity), $5.0 \mathrm{mg}$; riboflavin, $9.9 \mathrm{mg}$; D-pantothenic acid, $29.7 \mathrm{mg}$; niacin, $44.0 \mathrm{mg}$; vitamin B12, $0.04 \mathrm{mg}$; D-biotin, $0.85 \mathrm{mg}$; folic acid, $7.16 \mathrm{mg}$; thiamine, $8.8 \mathrm{mg}$; pyridoxine, $4.5 \mathrm{mg}$; chromium (chromium propionate), $0.40 \mathrm{mg}$; $\mathrm{Zn}\left(20 \% \mathrm{ZnSO}_{4}\right.$, $30 \%$ zinc oxide, and 50\% Mintrex Zn, Novus, St. Louis, MO), $150 \mathrm{mg}$; $\mathrm{Cu}\left(50 \% \mathrm{CuSo}_{4}\right.$ and 50\% Mintrex Cu, Novus, St. Louis, MO), $15 \mathrm{mg}$; $\mathrm{Fe}\left(\mathrm{FeSO}_{4}\right), 100 \mathrm{mg} ; \mathrm{Mn}\left(50 \% \mathrm{MnSO}_{4}\right.$ and $50 \%$ Mintrex Mn, Novus, St. Louis, MO), $50 \mathrm{mg}$; I (ethylenediamine dihydriodide), $0.4 \mathrm{mg}$; and $\mathrm{Se}\left(50 \% \mathrm{Na}_{2} \mathrm{Se}\right.$ and $50 \%$ organic $\left.\left.\mathrm{Se}\right)\right), 0.30 \mathrm{mg}$. Phytase was provided as Axtra (Danisco Animal Nutrition, Wiltshire, United Kingdom) and added 374 phytase units $/ \mathrm{kg}$ diet.

${ }^{4}$ Calculated from NRC (2012).

${ }^{5} \mathrm{M}+\mathrm{C}=$ methionine + cysteine.

pan; therefore, feed wastage was minimal. Feed intake was documented twice a day prior to each running of the feed system. Sows had ad libitum access to water throughout lactation.

\section{Animal Husbandry}

Sows were moved into the farrowing unit at $112 \pm 2 \mathrm{~d}$ of gestation. The sows were allocated to the experimental diets upon entry into the farrowing house. Sows were fed the allotted treatments from the time of entry into the farrowing house until weaning. Sows were housed in conventional farrowing stalls $(1.5 \times 2.1 \mathrm{~m})$ in an environmentally regulated commercial farrow to wean facility (18 to $24^{\circ} \mathrm{C}$ ) with lights on from 0600 to 1500 hours. Sows farrowed at $114 \pm 3 \mathrm{~d}$ of gestation and piglets were cross-fostered within treatment within $24 \mathrm{~h}$ of birth. In addition, the number of piglets (14 pigs) per sow was equalized across all treatment groups. Tails of piglets were clipped and $200 \mathrm{mg}$ of iron dextran was injected at $3 \mathrm{~d}$ of age. Male piglets were surgically castrated on day 3 . Piglets were not offered creep feed during the study, but did have access to water. In addition, rubber mats and heat lamps were provided as a source of supplemental heat to the piglets.

Sow and Piglet Performance. Sows were weighed at the time of entry into the farrowing unit (TruTest, Mineral Wells, TX and J\&H Automation, Gridley, IL) and on the day of weaning. Sow $48 \mathrm{~h}$ post-farrow BW was determined using the prediction equation: post-farrow weight, $\mathrm{kg}=[(112 \mathrm{~d}$ gestation weight, $\mathrm{kg}) \times 0.98)-20.81\left(R^{2}=0.93\right)$ (Greiner, unpublished data)]. The sow $48 \mathrm{~h} \mathrm{BW}$ equation was developed specifically for the genetic line. Sow weights were collected prior to farrowing along with variables such as total litter weight at birth, total number of pigs born, and sow BW $48 \mathrm{~h}$ after farrowing. A stepwise backward linear regression model was used to determine the sow $48 \mathrm{~h} \mathrm{BW}$ equation. The actual sow BW at $48 \mathrm{~h}$ was then crossed against the equation to confirm the equation.

In addition, piglet litter weights were recorded at $48 \mathrm{~h}$ of age and at weaning (Tru-Test, Mineral Wells, TX and J\&H Automation). Any mortalities and morbidities were recorded along with the piglet weights as the piglets were removed from trial. The removal weights and nursing days were calculated back into litter growth rate [(total litter wean weight - total starting litter weight + mortality weights $) /$ ((number of piglets weaned $\times$ lactation length $)+$ days moralities nursed)]. After weaning, sows were fed ad libitum a conventional gestation diet containing $3.17 \mathrm{Mcal} \mathrm{ME} / \mathrm{kg}$ and $0.61 \%$ total Lys. Sows were checked daily for signs of estrus using a mature boar beginning day 3 after weaning. Estrus was recorded when sows stood to be mounted by 
a boar, and days from weaning to estrus were also recorded. In addition, the number of sows bred within $10 \mathrm{~d}$ of weaning was recorded. Sows were provided $\sim 3.6 \mathrm{~kg}$ of gestation feed/d from weaning to mating. After mating, feeding levels were adjusted for visual body condition based on a farmspecific feeding scale that allowed body condition to be maintained at a 3.0 (scale of 0 to 5; National Pork Board Pork Quality Assurance, 2012) during the remainder of the gestation period. Weaning to mating interval, farrow to subsequent farrow interval and subsequent litter size, total born, born alive, stillborns, and mummies were recorded. For subsequent litter characteristics, only sows mated within $21 \mathrm{~d}$ postweaning and farrowing as a result of first mating were used.

\section{Diet Analysis}

The low Thr (46\% SID Thr:Lys) and high Thr (70\% SID Thr:Lys) diets were submitted for dietary AA analysis after manufacturing. Diets were submitted to Ajinomoto Heartland, LLC (Chicago, IL) for AA and CP analysis (AOAC, 1995).

\section{Statistical Analysis}

Data were analyzed using procedures of SAS (SAS Inst, Inc., Cary, NC) and reported as LS means. The statistical model included treatment and parity. The sow was the experimental unit. Performance criteria were calculated using PROC MEANS and presented as LSMEANS + SEM. Evaluation of treatment effects was analyzed by ANOVA using MIXED procedures. Polynomial coefficients were used to determine linear effects on increasing SID Thr:Lys ratio. A value of $P<0.05$ was considered significant. Broken-line models were fitted to daily litter gain to further estimate SID Thr:Lys requirements using NLMIXED in SAS (Robbins et al., 2006; Pesti et al., 2009). Statistical models fitted to the data included a broken-line linear (BLL) ascending model and a broken-line quadratic (BLQ) ascending model. For the BLL ascending model: $y_{i j}=L_{\mathrm{BLL}}+U_{l}\left(R_{\mathrm{BLL}}-X_{i}\right)+b_{j}+e_{i j}$, for $X_{i}<R_{\mathrm{BLL}}, y_{i j}=L_{\mathrm{BLL}}+b_{j}+e_{i j}$ for $X_{i} \geq R_{\mathrm{BLL}}$. For the BLQ ascending model: $y_{i j}=L_{\mathrm{BLQ}}+U_{q}\left(R_{\mathrm{BLQ}}-\right.$ $\left.X_{i}\right) 2+b_{j}+e_{i j}$, for $X_{i}<R_{\mathrm{BLQ}}, y_{i j}=L_{\mathrm{BLQ}}+b_{j}+e_{i j}$ for $X_{i}$ $\geq R_{\mathrm{BLQ}}$. In these equations, $y_{i j}$ is the response of the sow in the block $j$ assigned to treatment $i, X_{i}$ is the SID Thr level of the $i$ th dietary treatment, and $L_{\mathrm{BLL}}$ and $L_{\mathrm{BLQ}}$ indicate the unknown maximum response to the dietary treatments to reach plateau using the BLL and BLQ models. $R_{\mathrm{BLL}}$ and $R_{\mathrm{BLQ}}$ are the unknown minimum levels of the SID Thr required to reach plateau using the BLL and BLQ models. Furthermore, $b_{j}$ is the random blocking effect of the parameter associated with the $j$ th block and $e_{i j}$ is the random error associated with the sow in the $j$ th block that received the $i$ th treatment.

Statistical models were compared using maximum likelihood-based fit criteria (Schwarz Bayesian information criterion (Milliken and Johnson, 2009)). The best-fitting model was reported with a $95 \%$ confidence interval.

\section{RESULTS}

The average lactation period of the sow was 20 d. Sows lost on average $6.1 \%$ of their BW with an ADFI of $4.73 \mathrm{~kg} / \mathrm{d}$. Sow weaning-to-estrus interval was $5.00 \mathrm{~d}$. Sows started the trial with an average of 13.0 piglets/sow and weaned 12.0 piglets/sow with a piglet ADG of $0.330 \mathrm{~kg} / \mathrm{d}$ and a daily litter growth rate of $2.72 \mathrm{~kg} / \mathrm{d}$. Forty-two sows did not complete the lactation period of the study. Reasons for removal included mortality, illness, weaning 7 or fewer piglets as this can cause early onset of estrus, or feed valve failure resulting in incorrect feed delivery.

Diet analysis demonstrated that the planned ratios were similar to the actual ratios (Table 2). Sow ADFI (Table 3) peaked $(P<0.05$; quadratic) when the SID Thr:Lys ratio was at $68 \%$. Sow BW loss improved quadratically $(P<0.05)$ as the SID Thr:Lys ratio increased above $60 \%$. There was no difference between treatment groups for the weaning-to-estrus interval $(P>0.05)$. However, the percentage of sows bred by $10 \mathrm{~d}$ was quadratically improved $(P=0.02)$

Table 2. Planned vs. analyzed CP, total Lys, and total Thr values of the low (52) and high (84) standardized illeal digestible (SID) Thr:Lys diets

\begin{tabular}{llr}
\hline \hline & \multicolumn{2}{c}{ SID Thr:Lys ratio } \\
\cline { 2 - 3 } Item & 52 & 84 \\
\hline Planned & 16.25 & 16.41 \\
CP, $\%$ & 1.03 & 1.03 \\
Total Lys, \% & 0.56 & 0.80 \\
Total Thr, \% & 0.54 & 0.78 \\
Total Thr:Lys & 0.46 & 0.70 \\
SID Thr:Lys & & \\
Analyzed & & \\
CP, $\%$ & 16.35 & 16.88 \\
Total Lys & 1.05 & 0.97 \\
Total Thr & 0.62 & 0.78 \\
Total Thr:Lys & 0.59 & 0.80 \\
SID Thr:Lys & 0.52 & 0.84 \\
\hline
\end{tabular}

${ }^{1}$ Analyzed values represented as total percentage in the diet. 
Table 3. Evaluation of lactating primiparous and multiparous sow and litter performance as dietary standardized ileal digestible (SID) Threonine:Lys ratio increases in sow lactation diets

\begin{tabular}{|c|c|c|c|c|c|c|c|c|}
\hline \multirow[b]{2}{*}{ Parameter } & \multicolumn{4}{|c|}{ SID Thr:Lys ratio } & \multirow[b]{2}{*}{84.0} & \multirow[b]{2}{*}{ SEM } & \multirow[b]{2}{*}{$\operatorname{Trt}^{2}$} & \multirow[b]{2}{*}{ Quadratic } \\
\hline & 52.0 & 60.0 & 68.0 & 76.0 & & & & \\
\hline \multicolumn{9}{|l|}{ Sow } \\
\hline Number of sows & 52 & 62 & 61 & 57 & 58 & & & \\
\hline SID Lys intake, g/d & 48.7 & 52.6 & 46.7 & 44.8 & 44.6 & & & \\
\hline Total Thr intake, g/d & 29.3 & 30.1 & 32.7 & 33.7 & 35.9 & & & \\
\hline SID Thr intake, g/d & 21.8 & 23.8 & 27.6 & 29.6 & 32.7 & 0.60 & 0.001 & 0.001 \\
\hline ADFI, kg & 4.73 & 4.57 & 4.67 & 4.55 & 4.60 & 0.10 & 0.55 & 0.001 \\
\hline Sow $48 \mathrm{~h}$ weight, $\mathrm{kg}$ & 210.35 & 216.09 & 212.84 & 214.64 & 210.07 & 4.81 & 0.84 & 0.89 \\
\hline Sow wean weight, $\mathrm{kg}$ & 196.02 & 199.50 & 197.05 & 200.79 & 196.89 & 4.53 & 0.92 & 0.82 \\
\hline Weight change, $\%$ & -6.44 & -7.89 & -7.35 & -6.19 & -5.81 & 1.00 & 0.45 & 0.004 \\
\hline Bred by $10 \mathrm{~d}, \%$ & 90.3 & 95.6 & 91.8 & 87.7 & 84.3 & 0.03 & 0.05 & 0.02 \\
\hline Weaning-to-estrus, $\mathrm{d}$ & 5.42 & 4.42 & 4.55 & 4.46 & 4.84 & 0.30 & 0.07 & 0.12 \\
\hline Subsequent total born, $n$ & 12.67 & 13.90 & 14.45 & 12.86 & 12.99 & 0.56 & 0.05 & 0.02 \\
\hline \multicolumn{9}{|l|}{ Piglet } \\
\hline Number of piglets started/sow, $n$ & 13.19 & 13.50 & 13.54 & 13.68 & 13.75 & 0.21 & 0.29 & $>0.10$ \\
\hline Number of piglets weaned/sow, $n$ & 11.71 & 11.88 & 12.29 & 12.13 & 11.95 & 0.29 & 0.56 & $>0.10$ \\
\hline Pig ADG, kg/d & 0.223 & 0.219 & 0.223 & 0.224 & 0.219 & 0.005 & 0.87 & 0.001 \\
\hline Daily litter ADG, $\mathrm{kg} / \mathrm{d}$ & 2.67 & 2.67 & 2.78 & 2.77 & 2.68 & 0.077 & 0.63 & 0.001 \\
\hline Removal, \% & 11.01 & 12.12 & 9.18 & 11.55 & 13.27 & 1.63 & 0.36 & $>0.10$ \\
\hline
\end{tabular}

as the SID Thr:Lys ratio increased to $60 \%$, but then declined as the ratio increased. Increasing the SID Thr:Lys ratio resulted in a quadratic improvement $(P$ $=0.02$ ) in subsequent total born as the SID Thr:Lys ratio increased to $68 \%$. Piglet average daily gain improved quadratically $(P<0.05)$ up to an SID Thr:Lys ratio of $74 \%$. The optimal SID Thr:Lys ratio for litter ADG was $68 \%(P=0.001)$. The broken-line regression analysis indicated that the optimal SID Thr:Lys ratio daily piglet growth was $65 \%$ (Figure 1 ).

\section{DISCUSSION}

The analyzed dietary analysis was slightly higher than the calculated values. Therefore, all discussion will be based on the analyzed values and not the calculated. Feed intake in this study was allocated to $5.45 \mathrm{~kg} / \mathrm{d}$ to allow sows to consume above the NRC (2012) calculated requirements, but to minimize the variation in digestible Lys intake associated with sow ad libitum feed intake variation. Based on the litter growth rate of $2.70 \mathrm{~kg} / \mathrm{d}$, NRC (2012) SID Lys requirements for a sow per day would be $49 \mathrm{~g}$. Research conducted by Touchette (1998) indicated that the average SID Lys requirement for the sows in this study would be $\sim 51 \mathrm{~g}$ SID Lys/d. For sows whose litters are 2 SDs higher in daily growth rate, the estimated SID Lys per day requirement would be $55 \mathrm{~g}$ and the sows whose litters are 2 SDs below the average would require $48 \mathrm{~g} / \mathrm{d}$. Research conducted on high producing sows with higher litter growth rates indicated that $62 \mathrm{~g}$ of SID Lys/d is required for optimal sow reproduction (Greiner et al. 2009, 2011). In this study, sows consumed on average $56 \mathrm{~g}$ of SID Lys/d. Therefore, according to Greiner et al., the sows in this study were consuming below the expected Lys requirement to support litter growth and any responses associated with sow and piglet performance are not associated with any variation in the digestible lysine intake.

Additionally, Lys and Thr requirements could be determined based on anticipated milk output. Estimated CP milk output can be calculated using the following equation: $[(0.0257 \times$ mean litter gain $(\mathrm{g}))+0.42 \times$ litter size $)] \times 6.38(\mathrm{NRC}, 2012)$. From milk CP output, calculations can then be made on gram of AAs per day. Milk has $7.01 \mathrm{~g} \mathrm{Lys} / 100 \mathrm{~g}$ $\mathrm{CP}$ and Thr is $61 \mathrm{~g} / 100 \mathrm{~g}$ of Lys (NRC, 2012). From these values and using the average daily litter gain and litter size from this study, it is estimated that the average milk AA output during this study was $33.4 \mathrm{~g} \mathrm{Lys} / \mathrm{d}$ and $20.4 \mathrm{~g}$ Thr/d. By using the efficiency values for SID AA utilization $(0.67$ and 0.76 for Lys and Thr, respectively (NRC, 2012)) of the mammary gland for the lactating sow, the estimated daily requirements for SID Lys and Thr would be $49.9 \mathrm{~g}$ SID Lys/d and 26.0 g SID Thr/d. Based on the milk output calculation, sows in this study were consuming above the estimated Lys requirement for milk output and the test threonine levels in this study were above and below the estimated requirements for the sows. 


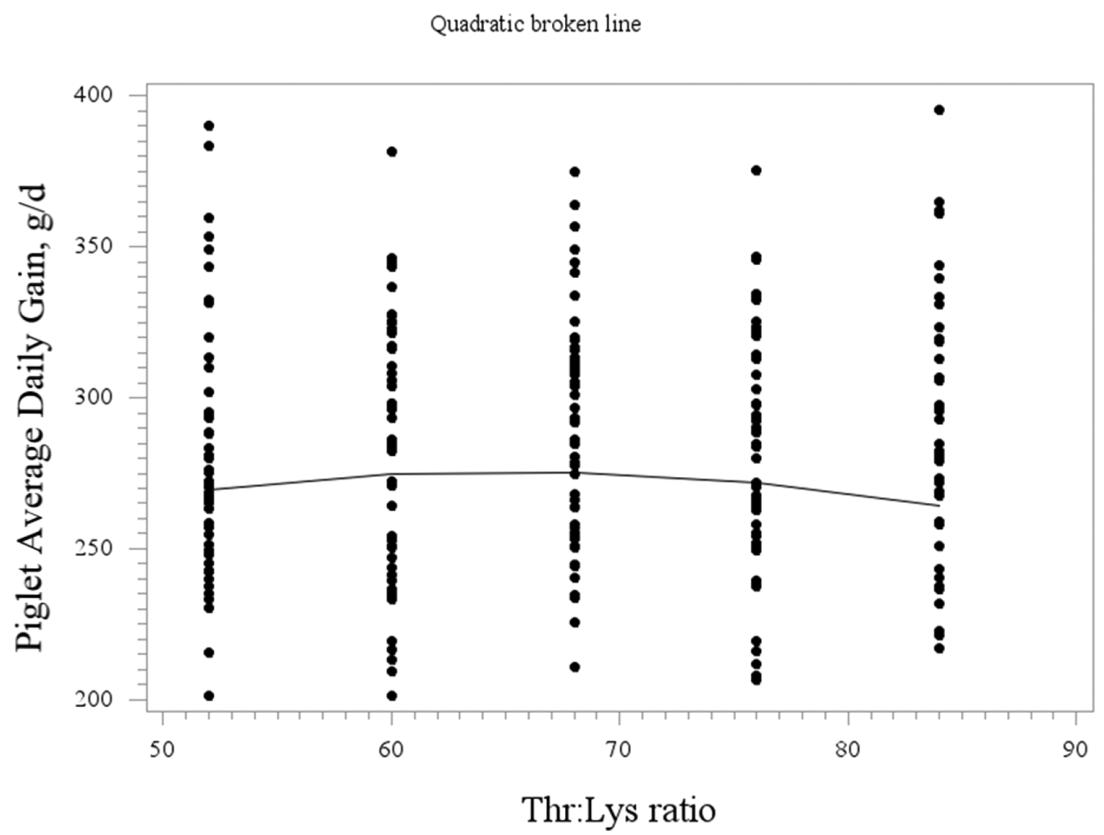

Figure 1. Quadratic broken-line regression analysis for the standardized ileal digestible (SID) Thr:Lys ratio on lactating primiparous and multiparous sow average daily piglet gain $(\mathrm{g} / \mathrm{d})$. The Bayesian information criterion for the quadratic analysis was $3,141.6$ with the ratio estimate of 65 .

In this study, there were parity effects on parameters such as ADFI, weight change percentage, weaning-to-estrus interval, and litter performance. However, there were no parity $\times$ treatment interactions, indicating that the optimal SID Thr:Lys ratio of $65 \%$ is the same for all animals. This finding was not predictable. It would be assumed that a young female would have a higher need for Thr due to the higher rate of protein synthesis required for structural growth. In this study, sows lost weight during lactation. It is likely that a portion of the threonine needs for growth came from the metabolism of lean mass of the younger parity sow rather than the feed; thereby, causing no differences in the feed allowance of Thr associated across parities.

The sow BW loss in this study was at a higher percentage than that predicted by the NRC equations (2012) based on litter size and piglet growth rate. Overall, sow BW loss was improved as the SID Thr:Lys ratio increased in the study. Threonine has a key role in protein synthesis as well as protein digestion. Threonine can be converted to glycine and serine, which are key metabolites for protein synthesis. Threonine is a key AA for digestion, such as a precursor for digestive enzymes such as chymotrypsinogen and trypsinogen (Smith, 1966) and improving mucosal villi length (Law et al., 2007). The improvement in digestion and potentially intestinal mass (Stoll, 2006) all result in the improvement in sow BW loss as the SID Thr concentration increased. The reduction in $\mathrm{BW}$ loss has been previously associated with improvements in sow reproduction such as an improvement in subsequent total born piglets (Thaker and Bilkei, 2005).

NRC (2012) recommends $31.1 \mathrm{~g}$ SID Thr/d for the mature female that is nursing piglets gaining $\sim 230 \mathrm{~g} / \mathrm{d}$. In this study, piglet ADG was $220 \mathrm{~g} / \mathrm{d}$. Sows in this study consumed between 21 and $33 \mathrm{~g}$ SID Thr/d. This calculation would indicate that the diets fed in this study were above and below the NRC recommendation. The results of the study further support that the diets were fed around the sow's requirement due to the biological responses described in the results. However, in this study, sow reproduction or litter growth rate improvements corresponded with daily SID Thr intake closer to 27 g. Subsequent sow reproduction was optimized when SID Thr:Lys ratio was at $68 \%$ or SID Thr was at $27.6 \mathrm{~g} / \mathrm{d}$. Broken-line analysis determined the SID Thr:Lys ratio optimal for piglet daily litter gain to be $65 \%$. The findings from this study suggest that the actual daily threonine requirement is lower than that the NRC (2012) recommendation for daily SID Thr, but a higher SID Thr:Lys ratio is needed.

Using the litter growth rate data from this study and the NRC (2012) equations to predict the SID Thr and Lys requirements, the estimated SID Trp:Lys ratio for the sows in this study to optimize litter growth rate would be 62.8. In this study, there were significant differences in the percent of sows bred in $10 \mathrm{~d}(2 \%$ change), subsequent total born pigs (1.8 pigs/sow), sow BW loss (11\% change), and litter growth rate response $(0.10 \mathrm{~kg} / \mathrm{d})$ associated with animals being above and below the NRC estimate; thereby concluding that 
the SID Thr:Lys requirement for litter growth rate is 65 and subsequent reproduction is 68 .

Conflict of interest statement. None declared.

\section{LITERATURE CITED}

AOAC. 1995. Official methods of analysis. 16th ed., Washington, DC: Association of Official Analytical Chemists.

Cooper, D. R., J. F. Patience, R. T. Zijlstra, and M. Rademacher. 2001. Effect of nutrient intake in lactation on sow performance: determining the threonine requirement of the high-producing lactating sow. J. Anim. Sci. 79:2378-2387. doi: $10.2527 / 2001.7992378 x$

Deen, J., and J. Xue. 1999. Sow mortality in the US: an industry-wide perspective. University of Minnesota Digital Conservancy, St Paul, MN. Available from http:// hdl.handle.net/11299/148140 (Accessed 10 September 2018.)

Greiner, L., J. Soto, J. Connor, G. Allee, J. Usry, and N. Williams. 2009. The evaluation of feeding lactating sows on grams of lysine compared to percent of lysine in the diet. J. Anim. Sci. 87 (e-Suppl. 3):16 (Abstract).

Greiner, L., J. Usry, C. Neill, N. Williams, J. Soto, J. Connor, and G. Allee. 2011. The evaluation of lysine requirements in third parity sow diets. J. Anim. Sci. 89 (e-Suppl. 2):64 (Abstract)

Law, G. K., R. F. Bertolo, A. Adjiri-Awere, P. B. Pencharz, and R. O. Ball. 2007. Adequate oral threonine is critical for mucin production and gut function in neonatal piglets. Am. J. Physiol. Gastrointest. Liver Physiol. 292:G1293G1301. doi:10.1152/ajpgi.00221.2006

Milliken, G. A., and D. E. Johnson. 2009. Analysis of messy data: designed experiments. 2nd ed. Vol. 1.Boca Raton, FL: CRC Press.
National Pork Board. 2012. Pork quality assurance plus. Des Moines, IA: National Pork Board.

Nielsen, S. E., A. R. Kristensen, and V. Aa. Moustsen. 2018. Litter size of Danish crossbred sows increased without changes in the sow body dimensions over a thirteen year period. Livest. Sci. 209:73-76. doi:10.1016/j. livsci.2018.01.015

NRC. 1998. Nutrient requirements of swine. 10th rev. ed. Washington, DC: National Academies Press.

NRC. 2012. Nutrient requirements of swine. 11th rev. ed. Washington, DC: National Academies Press.

Pesti, G. M., D. Vedenov, J. A. Cason, and L. Billard. 2009. A comparison of methods to estimate nutritional requirements from experimental data. Br. Poult. Sci. 50:16-32. doi:10.1080/00071660802530639

Robbins, K. R., A. M. Saxton, and L. L. Southern. 2006. Estimation of nutrient requirements using broken-line regression analysis. J. Anim. Sci. 84 (Suppl.):E155-E165. doi:10.2527/2006.8413_supple155x

Schneider, J. D., J. L. Nelssen, M. D. Tokach, S. S. Dritz, R. D. Goodband, and J. M. DeRouchey. 2005. Determining the threonine requirement of the lactating sow. Kansas Agric. Exp. Station Res. Rep. 2005:24-29.

Smith, M. H. 1966. The amino acid composition of proteins. J. Theoret. Biol. 13:261-281.

Stalder, K. J. 2018. 2017 Pork industry productivity analysis. Des Moines, IA: National Pork Board.

Stoll, B. 2006. Intestinal uptake and metabolism of threonine: nutritional impact. Adv. Pork Prod. 17:257-263.

Thaker, M. Y. C., and G. Bilkei. 2005. Lactation weight loss influences subsequent reproductive performance of sows. Anim. Reproduct. Sci.. 88:309-318. doi:10.1016/j. anireprosci.2004.10.001

Touchette, K. J., G. L. Allee, M. D. Newcomb, and R. D. Boyd. 1998. The lysine requirement of lactating primiparous sows. J. Anim. Sci. 76:1091-1097. doi:10.2527/1998.7641091x 